\title{
Airway and systemic eosinophilia in asthma: does site matter?
}

\author{
Konstantinos Kostikas, Eleftherios Zervas and Mina Gaga
}

Affiliations: 7th Respiratory Medicine Dept and Asthma Centre, Athens Chest Hospital, Athens, Greece.

Correspondence: Mina Gaga, 7th Respiratory Medicine Dept and Asthma Centre, Athens Chest Hospital Sotiria, 152 Mesogion Ave, Athens 11527, Greece. E-mail: minagagadayahoo.com

0 @ERSpublications

Assessing eosinophilia in asthma is pivotal: diffuse or localised predicts poor control and increased future risk http://ow.ly/vdUhl

Asthma is a complex chronic inflammatory disorder of the airways. It involves several cell types and cellular elements, is associated with airway hyperresponsiveness, and is characterised by recurrent episodes of wheezing, breathlessness and cough. These symptoms restrict the patients' daily activities, impact on their quality of life and, if severe and untreated, may lead to hospitalisation and death [1].

Asthma needs to be monitored and controlled, and treatment schemes follow a step-wise approach, based initially on the level of severity and then on the level of control. The assessment of asthma control includes several clinical parameters such as frequency and severity of symptoms, impact on daily activity, and use of medication. All these parameters involve the patients' perception and are often subjective [1]. However, monitoring of airway inflammation for the evaluation of asthma control may provide objective markers of assessment but requires time and financial and technological resources. Therefore, the use of inflammatory markers in the monitoring of asthma remains highly controversial.

While the evaluation and monitoring of airway inflammation status does not seem warranted in everyday clinical management of controlled mild-to-moderate asthma, the assessment of the inflammatory phenotype is required in severe asthma that is resistant to conventional treatment, while monitoring of inflammation may help control and reduce exacerbation rates [2].

Several attempts have been made to classify patients with treatment-resistant asthma into distinct phenotypes, often involving indices of airways inflammation [3-5]. Early reports suggested that patients with more severe asthma had increased eosinophil [6] and/or neutrophil [7] counts in induced sputum and in bronchial biopsies. Although some severe asthma phenotypes and particularly inhaled corticosteroid (ICS)-resistant asthma have been associated with the presence of neutrophil predominance in sputum $[6,8]$, many patients with severe refractory asthma have increased sputum eosinophil counts [9]. In those patients, eosinophilia persists despite intensified ICS treatment and may be related to local corticosteroid resistance. The importance of monitoring and managing asthma on the basis of biomarkers is highlighted in patients with discordance between symptoms and airway inflammation: in the cluster analysis by HALDAR et al. [10], patients with discordance between symptoms and eosinophilic airway inflammation were the ones who benefited more from inflammation-guided management. Asthma management based on sputum eosinophil counts has been related to significant reduction in exacerbations compared to conventional treatment [11,12], with a more prominent effect on eosinophilic exacerbations [12]. The recent European Respiratory Society/American Thoracic Society guidelines on severe asthma suggest the addition of sputum eosinophil counts only if performed in centres with experience in severe asthma, a dedicated laboratory and a specific sputum induction protocol $[13,14]$.

Received: Feb 192014 | Accepted: March 052014

Conflict of interest: None declared.

Copyright @ERS 2014 
Surrogate markers of airways inflammation have long been investigated. The exhaled nitric oxide fraction ( $\mathrm{FeNO}$ ) is a possible surrogate biomarker of airways eosinophilia but is not currently recommended for routine severe asthma management [13] due to the presence of significant confounders in its interpretation and the contradictory results in clinical trials [15]. FeNO was not more sensitive than clinical assessment by available questionnaires [16] or sputum eosinophilia [17] in predicting loss of asthma control. A recent study from the US National Heart, Lung and Blood Institute's Severe Asthma Research Program has shown that, despite some statistically significant associations, candidate surrogate biomarkers (including FeNO, IgE, blood eosinophil and neutrophil counts, and forced expiratory volume in $1 \mathrm{~s} \%$ predicted) failed to predict sputum cell count percentages accurately [18]. In a large cohort of 508 patients, blood eosinophils $(>220$ cells per $\mathrm{mm}^{3}$ or $>3 \%$ ) presented a modest predictive accuracy for sputum eosinophilia (areas under the curve in receiver operating curve analysis of 0.79 and 0.81 , respectively), whereas blood neutrophils were not predictive of sputum neutrophilia [19]. Data on the relationship between eosinophilic inflammation and patients' symptoms and asthma control remain controversial [20].

In this issue of the European Respiratory Journal (ERJ), SCHLEICH et al. [21] provide evidence that may improve our understanding of the associations between the site of eosinophilia and clinical parameters related to asthma control and future risk, as expressed by asthma exacerbation rates. The authors evaluated these associations in a retrospective cohort of 508 asthma patients with successful sputum induction and further validated their results in a prospective cohort of 250 consecutive "real-life" patients from the outpatient asthma clinic of a university hospital. The major finding of this study is the classification of patients, based on the site of eosinophilic inflammation, into four groups with distinct clinical characteristics: local/sputum eosinophilia (25\%), isolated systemic/blood eosinophilia (7\%), concordant/ diffuse sputum and blood eosinophilia (19\%), and absence of eosinophilic inflammation (49\%). Patients with peripheral eosinophilia did not differ phenotypically from those without eosinophilia, whereas patients with isolated sputum eosinophilia were characterised by lower lung function and higher bronchial hyperresponsiveness and FeNO compared to those without eosinophilia. Patients with diffuse eosinophilia had even worse lung function, worse asthma control and, most importantly, worse quality of life and higher exacerbation rate. Lower quality of life and higher exacerbation rate in patients with diffuse eosinophilia were also confirmed in the prospective cohort. It is important to stress that the four groups of asthma patients did not differ in terms of anti-inflammatory treatment intensity, as expressed by the prescribed doses of ICS. Interestingly, patients with concomitant local and systemic eosinophilia had more common nasal involvement (nasal polyposis or sinusitis) compared with all other groups and were more often atopic than the noneosinophilic patients. Important advantages of this study include the validation of the results in two separate cohorts (a retrospective and a prospective one) and the use of standard cut-off values for blood $\left(\geqslant 400\right.$ cells per $\left.\mathrm{mm}^{3}\right)$ and sputum $(\geqslant 3 \%)$ eosinophilia, whereas possible limitations include the recruitment of patients from a single university asthma clinic and the single time biomarker evaluation, given the fact that eosinophilic inflammation may well vary over time [22].

Is the study by SCHLEICH et al. [21] going to change our attitude towards asthma management and in what direction? The obvious answer is that treated patients with diffuse eosinophilia at a single time-point are at risk of future exacerbations and may therefore benefit from intensified treatment. But, at the same time, these patients presented worse asthma control, as expressed by increased scores on the Asthma Control Questionnaire, and would have required intensified treatment anyway. Therefore, is there an additional reason to implement eosinophil measurements in everyday clinical practice? Most of the patients in the study by SCHLEICH et al. [21] were already receiving intensive treatment: about two-thirds were on longacting $\beta$-agonists (LABAs) and ICS, with $\sim 30 \%$ receiving high-dose ICS, with a similar distribution between the four study groups. The fact that some patients presented sputum and/or blood eosinophilia despite ICS treatment reflects the need for management of eosinophilic inflammation beyond the effectiveness of ICS. In a recent analysis of a large randomised trial of omalizumab in patients with uncontrolled asthma on high-dose ICS and LABA, a reduction in asthma exacerbations was evident in patients with eosinophilic inflammation (increased FeNO or blood eosinophils) at baseline [23].

Refractory eosinophilic inflammation in patients with severe asthma has recently been addressed by interventions targeting Th2 cytokines, including interleukin (IL)-5, as well as IL-4 and IL-13. A recent trial showed that dupilumab, a human monoclonal antibody to the $\alpha$-subunit of the IL- 4 receptor, in patients with moderate-to-severe asthma with elevated blood or sputum eosinophil counts, was associated with fewer asthma exacerbations when LABAs and ICS were withdrawn, with improved lung function and reduced levels of Th2-associated inflammatory markers [24]. Two small trials of mepolizumab, a humanised monoclonal antibody against IL-5, had also shown beneficial effects in patients with refractory asthma with blood and/or sputum eosinophilia [25, 26]. The larger Dose Ranging Efficacy and Safety with Mepolizumab in Severe Asthma (DREAM) trial provided evidence of efficacy of mepolizumab in the reduction of asthma exacerbation in patients with severe eosinophilic asthma with an acceptable safety profile [27]. In this issue 
of the ERJ, ORTEGA et al. [28] report the results of a post hoc analysis of the DREAM trial and provide data showing that the reduction in exacerbations in asthmatic patients with refractory eosinophilia is observed irrespective of the presence of atopy (positive radioallergosorbent test to common aeroallergens) or total IgE levels. These data further support the need for the evaluation of the eosinophilic status of patients with treatment-resistant asthma, on top of the "traditional" assessments of atopic sensitisation.

The fact that asthma is no longer considered a single disease entity [29] strongly supports every effort to define the underlying inflammatory process. Since potential therapeutic options for noneosinophilic asthma are rather limited to date [5], the determination of the eosinophilic phenotype in treatment-resistant patients is a one-way option, especially in an era of emerging treatments targeting Th2 mediators. The data presented by SCHLEICH et al. [21] suggest that the site of eosinophilia does matter; whether diffuse or localised, it is a worse prognostic marker than the absence of eosinophilia, whereas the possible importance of isolated peripheral eosinophilia remains to be elucidated in future trials. To quote Sir Francis Bacon, "ipsa scientia potestas est" [30]: knowledge of the extent and the site of eosinophilic inflammation is power, the best available today in the management of difficult asthma.

\section{References}

1 Global Initiative for Asthma. Global Strategy for Asthma Management and Prevention 2012. www.ginasthma.org/ local/uploads/files/GINA_Report_March13.pdf

Wenzel S. Severe asthma in adults. Am J Respir Crit Care Med 2005; 172: 149-160.

Moore WC, Meyers DA, Wenzel SE, et al. Identification of asthma phenotypes using cluster analysis in the Severe Asthma Research Program. Am J Respir Crit Care Med 2010; 181: 315-323.

Wenzel S. Severe asthma: from characteristics to phenotypes to endotypes. Clin Exp Allergy 2012; 42: 650-658.

Wenzel SE. Asthma phenotypes: the evolution from clinical to molecular approaches. Nat Med 2012; 18: 716-725. Louis R, Lau LC, Bron AO, et al. The relationship between airways inflammation and asthma severity. Am J Respir Crit Care Med 2000; 161: 9-16.

7 Jatakanon A, Uasuf C, Maziak W, et al. Neutrophilic inflammation in severe persistent asthma. Am J Respir Crit Care Med 1999; 160: 1532-1539.

8 Moore WC, Hastie AT, Li X, et al. Sputum neutrophil counts are associated with more severe asthma phenotypes using cluster analysis. J Allergy Clin Immunol 2013; pii: S0091-6749(13)01563-7.

9 Tseliou E, Bessa V, Hillas G, et al. Exhaled nitric oxide and exhaled breath condensate $\mathrm{pH}$ in severe refractory asthma. Chest 2010; 138: 107-113.

10 Haldar P, Pavord ID, Shaw DE, et al. Cluster analysis and clinical asthma phenotypes. Am J Respir Crit Care Med 2008; 178: 218-224.

11 Green RH, Brightling CE, Woltmann G, et al. Analysis of induced sputum in adults with asthma: identification of subgroup with isolated sputum neutrophilia and poor response to inhaled corticosteroids. Thorax 2002; 57: 875-879.

12 Jayaram L, Pizzichini MM, Cook RJ, et al. Determining asthma treatment by monitoring sputum cell counts: effect on exacerbations. Eur Respir J 2006; 27: 483-494.

13 Chung KF, Wenzel SE, Brozek JL, et al. International ERS/ATS guidelines on definition, evaluation and treatment of severe asthma. Eur Respir J 2014; 43: 343-373.

14 Pizzichini E, Pizzichini MM, Leigh R, et al. Safety of sputum induction. Eur Respir J 2002; 20: Suppl., 9s-18s.

15 Kostikas K, Minas M, Papaioannou AI, et al. Exhaled nitric oxide in asthma in adults: the end is the beginning? Curr Med Chem 2011; 18: 1423-1431.

16 Kostikas K, Papaioannou AI, Tanou K, et al. Exhaled NO and exhaled breath condensate pH in the evaluation of asthma control. Respir Med 2011; 105: 526-532.

17 Quaedvlieg V, Sele J, Henket M, et al. Association between asthma control and bronchial hyperresponsiveness and airways inflammation: a cross-sectional study in daily practice. Clin Exp Allergy 2009; 39: 1822-1829.

18 Hastie AT, Moore WC, Li H, et al. Biomarker surrogates do not accurately predict sputum eosinophil and neutrophil percentages in asthmatic subjects. J Allergy Clin Immunol 2013; 132: 72-80.

19 Schleich FN, Manise M, Sele J, et al. Distribution of sputum cellular phenotype in a large asthma cohort: predicting factors for eosinophilic vs neutrophilic inflammation. BMC Pulm Med 2013; 13: 11.

20 Tillie-Leblond I, Montani D, Crestani B, et al. Relation between inflammation and symptoms in asthma. Allergy 2009; 64: 354-367.

21 Schleich FN, Chevremont A, Paulus V, et al. Importance of concomitant local and systemic eosinophilia in uncontrolled asthma. Eur Respir J 2014; 44: 97-108.

22 McGrath KW, Icitovic N, Boushey HA, et al. A large subgroup of mild-to-moderate asthma is persistently noneosinophilic. Am J Respir Crit Care Med 2012; 185: 612-619.

23 Hanania NA, Wenzel S, Rosen $\mathrm{K}$, et al. Exploring the effects of omalizumab in allergic asthma: an analysis of biomarkers in the EXTRA study. Am J Respir Crit Care Med 2013; 187: 804-811.

24 Wenzel S, Ford L, Pearlman D, et al. Dupilumab in persistent asthma with elevated eosinophil levels. N Engl J Med 2013; 368: 2455-2466.

25 Haldar P, Brightling CE, Hargadon B, et al. Mepolizumab and exacerbations of refractory eosinophilic asthma. N Engl J Med 2009; 360: 973-984.

26 Nair P, Pizzichini MM, Kjarsgaard M, et al. Mepolizumab for prednisone-dependent asthma with sputum eosinophilia. N Engl J Med 2009; 360: 985-993.

27 Pavord ID, Korn S, Howarth P, et al. Mepolizumab for severe eosinophilic asthma (DREAM): a multicentre, double-blind, placebo-controlled trial. Lancet 2012; 380: 651-659.

28 Ortega H, Chupp G, Bardin P, et al. The role of mepolizumab in atopic or non-atopic severe asthma with persistent eosinophilia. Eur Respir J 2014; 44: 239-241.

29 A plea to abandon asthma as a disease concept. Lancet 2006; 368: 705

30 Bacon F. Meditationes Sacrae, 1597. 